\title{
“Ellos contra nosotros", un Análisis Crítico de Discurso desde los sostenedores privados y los niños segregados en la Educación Chilena
}

\author{
Mónica Peña Ochoa \\ Universidad Diego Portales, Santiago, Chile. \\ Email:monica.pena@udp.cl
}

\begin{abstract}
Resumen $^{1}$ : Este trabajo analiza desde una perspectiva discursiva crítica, los discursos actuales sobre educación chilena y su relación con la discriminación, usando para ello dos fuentes discursivas: una columna de opinión escrita por el Secretario General de Colegios Particulares de Chile (CONACEP); y parte de los resultados del trabajo investigativo con niñas y niños de Octavo Básico de una escuela municipal del estrato socioeconómico bajo. Ambos casos, el primero desde la "libre elección" y cierto afectivismo y los segundos desde una lógica más territorial, dan cuenta de ciertos mecanismos de segregación que operan a nivel discriminatorio estructurados como un "ellos contra nosotros". Finalmente, se discute el carácter moral de la ideología del "buen estudiante” que impera a nivel hegemónico.
\end{abstract}

Palabras claves: Discriminación, educación, niños, análisis crítico de discurso.

\section{"Us vs. them", a Critical Discourse Analysis from the private providers and the children segregated in Chilean Education}

\begin{abstract}
The following paper analyzes some discourses on Chilean education today and their relation to segregation and discrimination, using for this purpose, two discursive sources: an opinion piece published in the Chilean newspaper El Mercurio on July 22, 2014 written by the Secretary General of Private Schools in Chile (CONACEP) that accounts the discursive discrimination is rooted in the Chilean educational model; and then part of the results of research with an 8th grade children of a municipal school from low socioeconomic status where discourses, although most hybrids, do not neglect the discursive repertoire of discrimination. The analysis found the us vs. them discourse, where segregation and discrimination operates and, from our perspective analysis of the hegemonic discourses that permeate the educational social practices at all levels.
\end{abstract} analysis.

Key words: Discrimination, education, children, critical discourse 


\section{“Nós contra eles”, uma Análise Crítica do Discurso de acordo com privados suportes e crianças segregados na Educação Chilena}

Resumo: Este trabalho analisa a partir de uma perspectiva discursiva crítica, os discursos atuais sobre educação chilena e sua relação com a discriminação, utilizando para o efeito, duas fontes discursivas: uma coluna de opinião escrita pelo Secretário-Geral das Escolas Privadas do Chile (CONACEP); e, em seguida, parte dos resultados de uma pesquisa com crianças de uma escola municipal de nível socioeconômico mais baixo. Ambos os casos, o primeiro desde a "livre escolha" e certo efetivismo e os segundos a partir de uma lógica territorial, permitem perceber certos mecanismos de segregação que operam num nível discriminatório estruturado como um "eles contra nós”. Por fim, discute-se o caráter moral da ideologia do "bom aluno” que é prevalecentemente hegemônico.

Palavras chaves: discriminação, educação, crianças, análise critico de discurso.

$* * *$

\section{Introducción: discursos hegemónicos sobre pobreza y vulnerabilidad}

El siguiente artículopretende dar cuenta de elementos comunes de algunos discursos sobre la educación chilena y su relación con la segregación y la discriminación, usando para ello dos fuentes discursivas: una columna de opinión publicada en el diario chileno el Mercurio el día 22 de julio del 2014 escrita por el Secretario General de Colegios Particulares de Chile, A.G (CONACEP), y parte de los resultados del trabajo investigativo con niñas y niños de Octavo Básico de una escuela municipal del estrato socioeconómico bajo (según ADIMARK). La tesis fundamental de este trabajo es que los discursos hegemónicos permean a distintos agentes de nuestra sociedad, y en este caso, ciertos discursos sobre la educación se vuelven dominantes, independientes de los contextos de donde provengan. El artículo presentará primero algunos elementos teóricos en relación a los estudios discursivos y su lugar en la educación chilena. Luego, presentaremos los dos discursos analizados desde una perspectiva discursiva crítica, y finalmente una discusión final que da cuenta de las relaciones de convergencia y divergencia entre las diferentes producciones discursivas presentadas, concentradas en el discurso del "buen estudiante". Creemos que este tipo de análisis es necesario hoy dada la discusión respecto de la llamada crisis educativa chilena, uno de los pocos espacios políticos que ha sido abierto a la discusión pública y ciudadana; y que a pesar de eso, se encuentra tremendamente amenazada por la dominancia a nivel de circulación y efecto de los discursos llamados hegemónicos.

Desde el año 1981 existe un proyecto de descentralización educacional en Chile, caracterizándose porque el Estado entrega fondos a privados para que se hagan cargo de entregar educación. En una primera etapa durante 
la dictadura militar específicamente- se asumió, siguiendo la ideología neoliberal desarrollada por Milton Friedman (1995), que la calidad del sistema se autorregularía a través del principio de la libre elección, propio del libre mercado. Una segunda etapa del procesose ha vivido con los gobiernos de la Concertación, donde se tuvieron que asumir las enormes brechas educativas que el sistema dejó, generando un sistema de subvenciones especiales orientadas a ayudar a aquellos estudiantes llamados "vulnerables” ya sea por razones económicas (Ley SEP en el año 2008 con cambios importantes el 2011)o por razones de salud (física y/o mental) a través de un diagnóstico especializado (Decreto 170 en el año 2010)² .

Las soluciones presentadas por la Concertación durante los primeros años que gobernó apuntaron a la disminución de las desigualdades optando por mantener el modelo, y mediante un sistema que mantuvo la subvención a la demanda se intentó mejorar las condiciones educativas. Un supuesto que es posible asumir para entender el lugar de los sujetos que se educan en Chile, es entender que para el Estado chileno los problemas educacionales no han sido del modelo o del sistema sinoque son de los individuos que se educan.Este aspecto puede entenderse a través de los procesos de “psicologización” de los fenómenos sociales y “psicopatologización” de las conductas fuera de la norma, en este caso dirigidos a los niños, niñas y adolescentes que se educan en Chile y a sus familias de origen que han sido estudiados desde una perspectiva crítica (por ejemplo Peña, 2013; Peña, 2011; Redondo, 2005;)también analizados por Parker, (2007); Burman, (1998) yRose, (1989). Se trata de procesos que cooperan en la perpetuación de la discriminación y segregación de los grupos de clases sociales más bajas, que son las más afectadas por las diferencias educativas.Las políticas públicas chilenas se han concentrado en la “vulnerabilidad social” como un fenómeno individual. Así, por ejemplo, el “Decreto 170” de subvención especial para necesidades educativas especiales se centra en la entrega de financiamiento a través de una vía que se considera objetiva como sería el diagnóstico médico (Peña, 2013). De esta manera, se omite la posibilidad de un modelo educativo inclusivo per - se, insistiendo en la alternativa de un modelo focalizado en individuos particulares con "necesidades especiales”. Podemos observar que se sigue la lógica particularista de los llamados "resultados educativos" que deben ser medibles y objetivos, convirtiendo a la medición de resultados más que en un termómetro para la decisión de las políticas públicas, un objetivo fundamental de la educación chilena (ver Popham, 1999; Redondo,2005).De esta forma, el aprendizaje, ha sido entendido como una "capacidad de aprendizaje”, es decir, una característica individual que se cultiva en el tiempo y que florecería mejor bajo ciertas condiciones naturales más que sociales, siendo desde una perspectiva discursiva una buena estrategia retórica para negar las diferencias y desigualdades de clase, género y etnia, entre otras. Las políticas públicas, según Shapiro (1989) pueden reflejar y reforzar prácticas hegemónicas, tendiendo a fracasar como proyectos, fundamentalmentepor no tomar en cuenta la complejidad social, económica y cultural, que sustenta todo proyecto político ${ }^{3}$. 
Las cifras respecto de los grupos pertenecientes al nivel socioeconómico más bajo son expresivas. No sólo los peores resultados académicos medidos de manera estándar por el SIMCE y la PSU se concentran en los estratos socioeconómicos bajos, sino que también la deserción escolar en educación media se concentra en estos grupos, indicador clave de lo que llamamos "fracaso escolar": las cifras varían entre el 7\% (CASEN, en línea, 2009), 8,7\% (CEOC, 2009); concentrándose casi toda en estratos socioeconómicos bajos (CASEN, en línea, 2009) al punto que esta cifra llegaría al 13,3\% de los niños de campamentos (Techo para Chile - UNICEF, 2011). Los “malos resultados” son una forma a través de la cual nos podemos aproximar a una definición de aprendizaje.

Aprendizaje sería un resultado objetivo que puede estandarizarse y ser medido por pruebas estandarizadas como el SIMCE y la PSU, que ocurre en la mente de los sujetos y que puede verse interrumpido por la vulnerabilidad social (según la Ley SEP) pero también por condiciones que afectan cierto funcionamiento normal (como lo dice el Decreto 170 a través de la idea “necesidades educativas especiales”). El aprendizaje también puede serentregado y depende en su entrega del profesor(a), entrega que puede ser evaluada a través de su desempeño medido en un historial y en la performance. Son las condiciones de los y las estudiantes sobre las cuales el programa de ayuda se concentra, es el hándicap y la falla la que levanta el problema. Esto implica que los aprendizajes, sin estas fallas debiesen expresarse de manera fluida, porque, una vez más, son los individuos los que presentan las habilidades para aprender.

Es en este contexto educativo, profundamente segregador, donde se es estudiante en Chile. Como veremos, los discursos políticos tienen correlatos a nivel de prácticas sociales, en el aula, pero también fuera de ella. A continuación, se explicará el contexto teórico - metodológico que nos permite hacer estas aseveraciones.

\section{Perspectiva metodológica: Un análisis discursivo crítico de la discriminación en educación}

Es posible considerar a la educación como un campo de lo político, en el sentido que lo plantea Mouffe(2007), es decir, una dimensión de antagonismo constitutivo de las sociedades humanas, donde los sujetos intervienen en la consideración de lo que una sociedad es o debería ser, y que se constituye y refleja en las diferencias y disensos de una comunidad. Desde esta perspectiva, es posible agregar que las diferencias, en las sociedades, no fluyen libremente, ni tienen todas, el mismo peso. Desde una perspectiva discursiva, podemos ver que el lenguaje encarna algunos elementos sociales generándose el constructo de "discurso", podemos entender que ciertos discursos circulan más y tienen mayores efectos que otros.Sin embargo, su circulación y efectos no dependen tanto del texto discursivo, sino fundamentalmente de los contextos socio - culturales y políticos que los conforman y consolidan (Fairclough, 1989; 1995). Un discurso es texto 
pero también contexto: los aspectos socio-históricos que sustentan al texto, así como también los aspectos lingüísticos, conversacionales, estéticos, entre otros.

El análisis crítico de discurso(ACD) se centra en los discursos que se producen y que circulan en una sociedad, discursos que pueden ser dominantes o "hegemónicos” provocando, dado su lugar de dominancia, más efectos sobre las prácticas sociales. Los discursos son declaraciones que construyen objetos por lo tanto, desde esta perspectiva no hay una diferencia radical entre dichos y prácticas, ya que el dicho en sí es una práctica social (Parker, 1992). Los textos entonces no son objetos comunicantes neutros. Son parte de las estructuras sociales que permiten o no ciertas prácticas sociales. Son los discursos hegemónicos los que pueden dar agencia a algunos sujetos, pero al mismo tiempo, constreñir a otros (Fairclough, 1989; 1995). El supuesto que manejamos es que es posible considerar que los discursos educativos chilenos, que circulan en nuestro sistema educativo y social en general, no sólo son la representación de cierto modelo económico y social imperante, sino que también constituyen ciertos límites y condiciones que inciden en las prácticas sociales de los individuos involucrados. Desde esta perspectiva, y dado que el ACD más que una metodología es un acercamiento teórico a los fenómenos, es posible considerar que hay aspectos profundamente discriminatorios en el modelo educativo chileno.

Es por lo anterior que utilizaremos la perspectiva discursiva crítica que propone RuthWodak $(2001,2012)$ ya que esta autora ha hecho un acabado trabajo acerca de las retóricas de la exclusión en los discursos oficiales y los medios masivos de comunicación, donde han sido analizados en profundidad la xenofobia, el antisemitismo, el nacionalismo, el etnicismo y el sexismo (Reisgl y Wodak, 2001). Wodak habla de "retóricas de la exclusión” para referirse a una serie de operaciones lingüísticas, siguiendo a las que ya en los años 70’s Van Dijk desde una perspectiva discursiva más cognitiva, había detectado (dominación, diferenciación, distancia, difusión, divergencia, despersonalización y discriminación cotidiana), y van a aparecer los aspectos contextuales, que son coherentes con uno de los objetivos del ACD: dar cuenta de cómo los discursos hegemónicos cuentan con circuitos también hegemónicos que garantizan su circulación, propagación y perpetuación (Wodak, 2001, 2012;Fairclough, 1995). No obstante, lo que nos interesa es cómo esos discursos discriminatorios no sólo se instalan en los círculos hegemónicos sino fundamentalmente, lo que la teoría de la hegemonía según Gramsci propone: cómo estos se hacen parte de los grupos subordinandos, o en palabras de Bourdieu, cómo se "reproducen” las violencias simbólicas impuestas en el día a día ${ }^{4}$.

La discriminación para Wodak (2012) implica la deprivación, a través de medios explícitos de poder simbólico implementados por las elites sociales, del acceso a participación, ciudadanía, medios, información, aprendizaje del lenguaje, posiciones de poder, ciertas organizaciones, trabajos, vivienda, educación. Esta deprivación se expresa a través de distintos dispo- 
sitivos discursivos - lingüísticos que Wodak (ibíd.) resume como nominaciones, predicaciones, argumentaciones, perspectivas o representaciones discursivas, así como la intensificación y la mitigación. Desde la perspectiva del ACD, dado su compromiso político con la emancipación,la discriminación puede y debe analizarse discursivamente ya que opera como una ideología;por tanto, opera también como práctica social. "Las opiniones discriminatorias, los estereotipos, los prejuicios y las creencias son producidas y reproducidas por medio del discurso y a través del discurso es que estas prácticas discriminatorias y excluyentes son preparadas, implementadas, justificadas y legitimadas” dice Wodak (2012: 406).

Es necesario agregar que los fines de este trabajo no son la generalización de un resultado a la manera de cierta investigación cualitativa más tradicional, ya que no se trabaja sobre una "muestra" que represente a un grupo. El ACD, al concentrarse en los discursos, tiene como pretensión identificar y reconocer propósitos, legitimaciones y deslegitimaciones sociales. Esta metodología busca esclarecer la posibilidad de los discursos de dar cuenta de acciones de carácter discursivo y que esas acciones, eventualmente, constituyen prácticas sociales (Van Leeuwen, 2008). Asimismo, hay otros elementos que dan fuerza al análisis crítico de discurso como metodología. Una de ellas son los conceptos de circulación y género de los discursos, que dan cuenta el primero de campo de efecto que puede tener un discurso (donde por ejemplo, algunos textos no necesitan la repetición ya que los medios de comunicación que los sustentan, en forma y fondo avalan el mensaje) y el de género, que da cuenta de las formas de actuar asociadas, implícitas pero reconocibles por sociedades que comparten los mismos semióticos (ver Fairclough, 2003)

\section{Análisis \\ Los sostenedores: el "ellos contra nosotros" y la fundamental segregación en la política chilena}

La Reforma Educacional que se ha discutido en el segundo Gobierno de la Presidenta Michelle Bachelet (2014- 2018) y que fue parte de su Programa Presidencial se sostiene en tres pilares: el fin al lucro, fin del copago y el fin de la selección en establecimientos que reciben fondos públicos. A la fecha es posible seleccionar a los y las estudiantes que van a matricularse a un establecimiento con los procedimientos que la institución considere válidos. La razón que da el Gobierno en su propuesta busca "promover y defender la libertad de elección para las familias respetando la diversidad de proyectos educativos que existen ${ }^{5}$, es decir, ya sean laicos, religiosos, artísticos, deportivos, entre otros. Por eso, a partir del 2016, se prohibirá que los establecimientos educacionales que reciben financiamiento público apliquen mecanismos de selección de estudiantes que generen discriminación arbitraria sobre la base de sus características socioeconómicas, étnicas o culturales.

Las principales reacciones frente a esta propuesta han venido de los representantes de los establecimientos particulares subvencionados. Du- 
rante los meses de mayo, junio y julio del 2014, en el diario chileno de circulación nacional "El Mercurio”, principal referente de la élite política conservadora del país (Cabalín, 2014), publicó 6 editoriales con referencia directa o indirecta al fin de la selección. Hemos elegido como tema de análisis a la selección ya que consideramos que apela directamente a la segregación y discriminación de estudiantes, y por la existencia de 13 columnas de opinión con similar tema (en este breve recuento no están contadas las cartas al Director referentes al tema). Del total de los 19 textos 4 defienden la selecciónatravés de argumentos relacionados con establecimientos de educación pública (en este caso, los llamados "Liceos de Excelencia" creados durante el Gobierno de la Alianza de partidos de la derecha de Sebastián Piñera). De todos los textos, sólo 3 columnas están a favor del fin de todo tipo de selección.

Para fines de este análisis, hemos escogido una de las columnas de opinión, publicada el día martes 22 de julio del 2014, escrita por Rodrigo Ketterer, el Secretario General de CONACEP ("Colegios Particulares de Chile, A.G.”) que se define como "una de las agrupaciones gremiales del sector educacional más importantes del país, que reúne a sostenedores de establecimientos educacionales particulares, tanto subvencionados como pagados. CONACEP está distribuida en todo el país...”6 .

La columna, de nueve párrafos, se titula de forma genérica como "Reflexiones sobre la reforma educacional”yse inicia con un "disclaimer", es decir, una operación discursiva que pretende morigerar la declaración posterior: "desde mi perspectiva, no hay ninguna duda que nuestro sistema educacional requiere profundas reformas para entregar un servicio de calidad” (Ketterer, 2014).

Los “disclaimers” buscan justificar una posterior defensa de la práctica discriminatoria a través de una presentación positiva de sí mismo (Wodak, 2012), en este caso, como abierto al cambio para la mejora de la calidad $^{7}$, cambio que ha sido avalado social y políticamente los últimos años.El "disclaimer”tiene también la función deseparar al autor de otros discursos que tendrían un "verdadero" prejuicio frente al cambio, situando su crítica como objetiva o factual.También es posible ver a lo largo del texto múltiples referencias o nominaciones que construyen una voz subjetiva particular que a la vez, se generaliza y apropia: son los padres, apoderados y profesores que aparecen como los representados por el autor, opacando su propia voz, y transformando el texto en una retórica de corte emocional - afectivo, dado lo fundamental de la familia y sus preocupaciones, como por ejemplo:“...ningún padre está disponible para que se realicen experimentos con la educación de sus hijos.” (Ketterer, 2014).

En esta cita vemos al menos dos elementos fundamentales: primero el uso del recurso retórico metafórico y eufemístico del "experimento" dirigido a "los hijos". Nadie prestaría a sus hijos para una acción que podría ser eventualmente dañina. Además, al usar el concepto de "experimento" para la reforma actual asume que la situación educativa actual está, naturalizada, 
es decir, vivimos en un proceso a - histórico(ver Fairclough, 1995).Es curiosa la elección del término, dado que no es ajeno a la forma que se describe el modelo educativo actual, también un experimento del "laboratorio neoliberal” que fue Chile durante los años de la Dictadura de Augusto Pinochet(Harvey, 2005).

La siguiente cita es el cuerpo del texto. Acá encontramos el núcleo de la argumentación. Llama la atención que este núcleo esté entre comillas, una operación que Wodak (2012) considera parte de las más comunes prácticas discursivas en relación a la discriminación, donde el hablante se posiciona de manera ambigua con lo que dice, se aleja al ponerlo como una cita no propia pero a la vez pretende generar un acercamiento con el lector al hacerlo coloquial, suponiendo que los lectores comparten también la significación del significante comillado.

"Es probable que a muchos haya sorprendido que, de las medidas del proyecto de ley, la que ha causado mayor rechazo entre los apoderados y profesores sea el fin de todo tipo de selección... La gran mayoría de los apoderados selecciona para sus hijos el colegio que según sus expectativas, les ofrece un "mejor ambiente” (sic), entendiendo por ello un establecimiento en el que existe la disciplina, orden, seguridad y adecuadas conductas de convivencia...” (Ketterer, 2014).

Nuevamente se parte con una operación de mitigación que da pie a la defensa de la selección sin decir nunca directamente que se la defiende, usando referenciales nuevamente, en nombre de padres y además profesores. En el siguiente punto, el cuerpo de toda la columna, pero también el más débil argumentativamente: el autor señala, usando nuevamente la operación referencial a padres y apoderados, pero esta vez no es el absoluto del “ningún padre”, ahora es una "gran mayoría” que dejaría a algunos elementos fuera. Para ello elige el eufemismo “mejor ambiente” que él mismo pone entre comillas.

En este caso ¿de qué se aleja? ¿Qué quiere mantener levemente mitigado, velado? El concepto de "buen ambiente", en su extrema vaguedad semántica, en Chile se usa para describir lugares de entretención donde los asistentes no serían de clases socioeconómicas bajas; pero también se asocia con buen clima (en el sentido “psicológico”, es decir, donde están dadas las condiciones para cierta convivencia). A la vez, que el autor diga "mejor ambiente" implica que hay ambientes peores, que en esta ambigüedad semántica pueden ser sociales o psicológicos, o ambos.

La idea de que hay mejores ambientes que otros se fundamenta cuando dice que "la gran mayoría de los apoderados", es decir, no todos, preferirían un mejor ambiente. En consecuencia hay un grupo de padres que no eligen ese mejor ambiente porque no tienen, siguiendo el razonamiento del autor, “expectativas” al respecto, por la razón que sea.La personalización del fenómeno de la elección, su psicologización extrema, la 
posible operación metonímica entre elección y deseo que supone el discurso neoliberalponen el problema en el individuo. De esta manera, quienes están en los colegios con “peor ambiente” podrían estar ahí porque de alguna manera eligieron un establecimiento en el que no existe la disciplina, ni el orden, ni la seguridad, ni las adecuadas conductas de convivencia que el autor describe como parte del establecimiento de "mejor ambiente”. Sería no sólo difícil tener que convivir para aquellos que eligen un buen ambiente con gente que elige un peor ambiente, sino que sería un ataque de carácter moral: el “ellos contra nosotros” es, según Wodak (2012), una de las bases más potentes de la argumentación discriminatoria.

Ahora bien, la columna describe a la seleccióncomo un fenómeno no educativo, sino más bien afectivo. La selección está por fuera del establecimiento, como si estos no tuvieran dispositivos de selección (p.ej. pruebas de selección de entrada, requisitos familiares o bien expulsión de estudiantes por baja de rendimiento o problemas disciplinarios) ni tampoco estaría en aspectos estructurales como el capital cultural o en las competencias sociales de las familias o adultos responsables para hacer elecciones. La elección es una competenciaindividual puesta en la decisión de los padres, por lo tanto el mal ambiente estaría asociado a niños y niñas hijos de padres que no eligen el buen ambiente. Ese mal ambiente, generado por la mala elección (o falta de elección) de algunos adultos, se mantendrá en otro lado, ya que el autor no ve como variable a intervenir el "ambiente" del establecimiento sino que su principal preocupación es la mantención del libre albedrío de los padres para elegir el tipo de ambiente que quieren para sus hijos. Este argumento, tan presente en el escenario educacional neoliberal, incluye además por omisión la falta de agencia de niños, niñas y jóvenes en el lugar de la decisión, poniendo de manera particularmente relevada el rol de los padres como entes universales y racionales respecto a las decisiones de los niños, cuyo lugar subjetivo principal pasa a ser el de la filiación más que el del aprendiz. Veamos un ejemplo concreto de cómo esto se sustenta políticamente y ya no sólo como una simple opinión personal, o en otras palabras, como hay un sustento estructural para este tipo de discursos:

Constitución 1980 Artículo 19 inciso $10^{\circ}$ “El derecho a la educación”.

La educación tiene por objeto el pleno desarrollo de la persona en las distintas etapas de su vida.

Los padres tienen el derecho preferente y el deber de educar a sus hijos. Corresponderá al Estado otorgar especial protección al ejercicio de este derecho.

La Constitución chilena del año 1980 delimita con claridad que el derecho a la educación no es para todos y todas, sino que es un derecho preferente de un sujeto delimitado: los padres.Esto conlleva al menos cuatro consecuencias: 
a) el sujeto de derecho son los padres, no los hijos.

b) de lo anterior se podría extrapolar que son los adultos pero no los menores de edad los sujetos de derecho.

c) no todos los adultos son los que tienen este derecho, son sólo aquellos que son padres.

d) no todos los niños pueden ser sujetos (intermedios) del derecho, sólo los que son hijos.

La frase se construye en base a una exclusión. Son los padres los que deciden sobre los hijos, por lo tanto se asume que todos y todas las que se educan en Chile son hijos y que hay padres en pleno derecho tomando decisiones, decisiones que como el sistema supone, siempre racionales. La consideración legal de que los padres tienen el derecho preferente y el deber de educar a sus hijos deja al Estado en lugar periférico otorgando especial protección al ejercicio de este derecho de los padres. La frase implica la creencia de que ningún padre querría lo peor para sus hijos, y da pie a que la educación y sus derechos correspondientes queden como una elección, supuesta siempre como correcta en tanto está puesta en un lazo que ni el Estado puede regular, que es el lazo entre padres e hijos. Si el Estado decide tomar este derecho como una obligación propia, no sólo lesionaría la libertad de las personas (consagrada en el mismo artículo 19) sino también la relación natural de saber y afecto que los padres (de manera universal) tienen para con sus hijos.

Ahora bien, una exclusión en sí misma no es una discriminación, a menos que lesione a una persona o un grupo de personas. En Chile, la definición de discriminación no es clara, situándose más bien del lado de la polémica, después de la promulgación de la “Ley Antidiscriminación” en el año 2012. Esta ley define discriminación como:

“toda distinción, exclusión o restricción que carezca de justificación razonable, efectuada por agentes del Estado o particulares, y que cause privación, perturbación o amenaza en el ejercicio legítimo de los derechos fundamentales establecidos en la Constitución Política de la República o en los tratados internacionales sobre derechos humanos ratificados por Chile y que se encuentren vigentes”. ${ }^{8}$

Como bien dice Muñoz (2013), es necesario detenerse sobre la “justificación razonable”, que según el autor corresponde a una "cláusula de textura abierta” (Ibid: 218) y es necesario preguntarse por el criterio para evaluar los actos denunciados bajo esta ley. El mismo autor propone que este criterio no es malo en sí, sino insuficiente: es necesario salir de la legalidad cómo se propone para no caer en el "positivismo ideológico", pero a la vez la simple “justificación razonable” es el indicio de la existencia de una conducta discriminatoria pero no la conducta discriminatoria en sí. En palabras de Muñoz, la discriminación debería de entenderse en función del mal social específico que intenta remediar la ley. Es por ello que la ley no debería sancionar simples conductas individuales avaladas por el mal comportamiento de un sujeto en particular sino que es necesario comprender 
que las decisiones deben tomarse -además- en relación a que la discriminación es un fenómeno social, avalado por grandes grupos sociales, a través de creencias compartidas, o como el ACD refiere, que se significa y propaga a través de ideologías imperantes.

En otras palabras, la discriminación se produce entre grupos aunque actuemos individualmente ya que la discriminación es un fenómeno social. En palabras de Muñoz (2013), el problema de nuestra ley antidiscriminación es que no toma en cuenta que "la razón por la que la discriminación es un fenómeno tan difícil de contrarrestar es precisamente porque la realidad es totalmente la contraria; más a menudo los sujetos que incurren en actos de discriminación lo hacen porque de su entorno concluyen que victimizar a ciertos grupos es fairgame(sic), está socialmente aceptado” (Muñoz, 2013: 5). En otras palabras, la estructura de la discriminación, como dice Van Dijk (2003) y Wodak (2012) se organiza como un “ellos contra nosotros”.

\section{Niños y niñas de una escuela "vulnerable": discriminación en primera persona}

Desde el primer semestre del año 2014 este equipo de investigación trabaja en una investigación-acción que tiene como objetivo analizar los productos de aprendizaje de adolescentes hombres y mujeres de octavo año básico de una comuna vulnerable y otra rural, desde una perspectiva discursiva crítica a través de una modalidad de trabajo de investigación acción participativa. Uno de los objetivos específicos es integrar a los sujetos participantes a través del reconocimiento crítico del lugar que tienen los jóvenes llamados “vulnerables” para lograr un posicionamiento contextual desde donde analizar los productos posteriormente.

Todos estos niños y niñas, viven en una de las comunas más pobres de Santiago, con un alto porcentaje de habitantes pertenecientes a los dos quintiles de más bajos ingresos, siendo esta escuela en particular una de las que atiende niños y niñas casi exclusivamente del nivel socioeconómico E (según ADIMARK) $^{9}$. Es un establecimiento municipal, por ende gratuito.A través de un juego de identidades sociales basado en Boltanski y Thevenot (1983) pudimos conocer mejor las “competencias" sociales de estos niños y niñas, que a través de fichas con datos sociales trascendentales para entender su contexto socio - educativo. Estos datos eran nombre (del que se podía suponer género), edad, comuna de domicilio, proveniencia del establecimiento educacional, ocupación del padre, ocupación de la madre, nivel de estudios de la madre, nivel de estudios del padre, y dos “distractores": hobby o pasatiempo y comida favorita.

Se les pidió a niños y niñas, en trabajo colectivo, que organizaran los juegos de 64 cartas en mazos según lo que a ellos les pareciera, al principio sin ninguna ayuda de los monitores.Luego, si decidían organizar los grupos por variables concretas como edad o tipo de comida, se les pedía más 
directivamente que buscaran otra forma de organizar. Es así que finalmente los 5 grupos funcionando pudieron organizar sus mazos en 3 grupos distintos dando cuenta de las diferencias sociales. Para confirmar estas decisiones y entender mejor el proceso de discusión crítica que los lleva a tomar sus decisiones se les pide que elijan dos de las variables mencionadas para adivinar en qué mazo va la “carta anónima”.Ahí ellos decidieron, a través de discusiones, si la ocupación del padre/madre o su nivel educacional, el domicilio o el tipo de escuelas eran las mejores formas de predecir el lugar socioeducativo de un sujeto ${ }^{10}$.

Uno de los resultados más decidores al respecto fue, que cuando se les pide que "bauticen” los mazos con las organizaciones de carácter social que ellos deciden, son los grupos de clases bajas los más discriminados a nivel lingüístico, tal como veremos en la Tabla 1.

Tabla 1: Nombres de los “mazos” según niveles socioeconómicos:

\begin{tabular}{|c|l|l|l|}
\hline Grupo & \multicolumn{1}{|c|}{ Nivel alto } & \multicolumn{1}{|c|}{ Nivel medio } & \multicolumn{1}{c|}{ Nivel bajo } \\
\hline 1 & "Los cuicos" & "Los miti - miti" & "Los shiwá" \\
\hline 2 & "Sueldo máximo" & "Sueldo mixto" & "Sueldo mínimo" \\
\hline 3 & "Cuicos" & "Clase media" & "Pobres" \\
\hline 4 & "Los bakanes" & "Los masomenenos" (sic) & "Los Shrek" \\
\hline 5 & "Generación" & "Los bobos" & "Los pavos" \\
\hline
\end{tabular}

Fuente: Elaboración Propia

“Los shiwá”, por ejemplo, alude a ciertas formas de habla del grupo referido. "Los shrek” se denominan así porque al igual que el ogro de la película “Shrek”, este grupo viviría “en el pantano”. Es posible dar cuenta que no hay ninguna referencia especialmente negativa hacia los grupos de nivel alto.

Vamos a usar como ejemplo de estos procesos discriminatorios lo que pasó en el grupo 5 (los grupos fueron armados al azar, por niños y niñas, y trabajaron en paralelo durante dos sesiones).En un inicio a los niños(as) les costó pensar en el estrato socioeconómico como una variable para agrupar las cartas. Las primeras divisiones que realizaron estaban relacionadas con género, edad y tipo de comida. A partir de las preguntas del monitor, comenzaron a surgir divisiones que utilizaban variables de carácter más social, que fueron expresadas en términos del nivel educacional de los padres, nivel ocupacional de los padres y la comuna de residencia.Las razones para bautizar a sus mazos se dieron como sigue: "Los pavos" son aquellos con padres que cursaron sólo hasta $8^{\circ}$ básico, "los bobos" son aquellos con padres que solo tienen educación media y los “generación” 
son los hijos de padres universitarios, y se llaman así porque son las generaciones futuras que podrán acceder a estudios superiores.

Durante la actividad aparecieron comentarios relacionados con la movilización estudiantil, en donde los niños señalaron no estar del todo de acuerdo con la gratuidad de la educación, ya que esta nueva política permitiría que “entrara cualquier niño al colegio”. Asimismo, debatieron respecto a si les gustaría cambiarse a un colegio pagado, y surgieron dos posiciones, una de los niños y niñas que dicen que no les gusta "el ambiente cuico", que no tiene "ni un brillo el colegio pagado"; mientras que la otra posición dice que sí les gustaría, porque les permitiría salir de la pobreza, una pobreza que está asentada, más que en el colegio, en la comuna donde habitan. Hay acuerdo en que todos quieren salir de la comuna en el futuro.

¿Quién es “cualquier niño”? La vaguedad de la expresión confirma la amplitud con que se expresa la segregación. Cualquier niño es exactamente lo contrario, no es cualquier niño, es un niño que no debería estar ahí. ¿”Cualquier niño” podrían ser ellos y ellas? la ambigüedad y ambivalencia discursiva da cuenta de lo complejo que es el tema de la experiencia de la pobreza y la segregación social vividos en primera persona. Efectivamente, “cualquier niño" puede ser eso, cualquiera, incluso ellos mismos, a través de una estrategia de distanciamiento.

Hay una intuición potente en los niños y niñascuando analizan el lugar de los colegios pagados: no es el colegio en sí mismo lo que desean, es más bien el cambio territorial el que ellos asocian a la salida de la pobreza: en una sociedad segregada como Santiago, por cierto que esto es un hecho realista, ya que la ciudad se organiza en torno a los niveles de ingreso de sus habitantes. No es la escuela ni la calidad de la educación el problema educativo en sí, es el territorio donde se habita, es la estigmatización que se porta. Esto es una solución de compromiso: no es el sujeto en sí el segregado, bastaría el cambio territorial, pero se omite que es imposible separar la experiencia subjetiva del habitar y el habitar del lugar que se habita.

Finalmente, es necesario insistir en que es absolutamente posible la convivencia de discursos distintos, opuestos inclusive,circulando y conformando nuestras prácticas sociales: el sujeto no solamente reproduce,también se queja einclusive resiste. Sin embargo, sin idealizar a los procesos de resistencia, no debemos de pasar por alto que la reproducción está presente de manera muy potente, configurando conflictos constantes en las prácticas sociales de los sujetos que no pueden excluirse por completo de la incesante repetición y circulación de los discursos hegemónicos (Abu-Lughod, 1990).

\section{Conclusiones}

Como es posible observar, no es necesario que los textos tengan violencia explícita, emociones exaltadas o sean emitidos por miembros que 
no pertenecen al grupo excluido para que existan discursos de la discriminación en la educación chilena. Los discursos discriminatorios operan de maneras mucho más sutiles.En este caso, de hecho, es el Estado el que detenta esta facultad, haciendo uso de los Wodak y Riesgl (2001) llamarían “la administración de la exclusión social”.Son las ideologías de carácter sexista, racista, homofóbica o clasista las que están a la base en las argumentaciones de los grupos que discriminan. La construcción de alteridades presentadas negativamente es la forma en que se actúa generalmente, desde una perspectiva retórica, pero también es posible constituir este otro disminuido desde la omisión que manipula así la existencia de grupos alternativos, sea considerándolos una excepción a la regla en el mejor de los casos, o bien una realidad anormal, ya sea criminal o patológica (Parker, 1992). Las posibles asociaciones de las clases más bajas con las características de falta de disciplina (y por cierto a la criminalidad) donde desde la teoría de los factores de riesgo se han incluso desarrollado políticas públicas educativas y de otras áreas, ha sido una constante en Latinoamérica.

¿Es la selección una forma de discriminación? Sí, porque su existencia implica que no existen las mismas oportunidades de desarrollo para todos los sujetos. Las oportunidades se reparten según nivel socioeconómico aunque se insista en retrucarlo como un asunto de insuficiencia moral puesta en el individuo con los conceptos de "ambiente" o “cualquier niño". Este “ellos contra nosotros” debe analizarse también desde el punto de vista del "nosotros" como detentores de ciertos derechos simplemente porque somos distintos, confundiendo privilegios con derechos. El "nosotros" es el representante de lo sano, lo moral, lo normal. El padre que elige el buen ambiente para su hijo es la figura estereotípica de esto. De alguna manera es posible ver que la figura clásica de la meritocracia como la del sujeto honesto, trabajador, que supera los escollos de la vida y no se distrae en su camino; tiene continuidad en este núcleo familiar que permite que esto ocurra, que da las condiciones subjetivas (y no estructurales) para que el sujeto se desarrolle a pesar del ambiente y las condiciones adversas que más que una imposición, son una elección.

Nuestro modelo educativo se basa en la creencia de que la diferencia individual es debida a una insuficiencia personal, intransferible, más parecida a una enfermedad que se hereda, que se potencia, que se cura y que también se previene, que es una inhabilidad para aprender pero que también podría ser una incapacidad para ser enseñado. La falta de "condiciones de aprendizaje” es un problema personal donde no sólo la pedagogía tiene poco que hacer: es una forma bastante sencilla de dirigir los esfuerzos públicos a individuos ignorando los efectos de las instituciones y la propia organización del modelo educativo en estos, quitando energía a la colectivización, democratización y participación en las comunidades escolares. Pero además de esto, en el modelo discriminatorio que nos encontramos operaunaideología del buen estudiante que da cuenta más que de habilidades de aprendizaje, de ciertas competencias morales, que en su reverso confunden pobreza con insuficiencia moral,conlo anti - social que se reflejaría en el colegio de mal ambiente, una cuna, semillero, o escuela de la 
criminalidad. Esto se acerca a lo que ya en 1984 habían desarrollado las investigadoras chilenas Assael y Neumann y que llamaron "cultura escolar del fracaso”. Las investigadoras, en su ya clásica etnografía, dan cuenta de las diversas prácticas de la escuela que van condenando prematuramente al fracaso a los estudiantes, lo que sería el reverso del "buen estudiante", aquel que según las autoras se acerca a la normalidad y la adaptación a las normas escolares. Esta fórmula, lejos de haberse desvanecido, se ha fortalecido, poniendo todas las posibilidades en el estudiante, y sus familias, que eligen o no ser buenos estudiantes.Se trata de una ideología porque, además de ser un sistema de ideas o creencias, como en parte la describe Van Dijk (2003), sino que también le da legitimidad a un grupo sobre otro, un grupo que puede situarse verticalmente sobre otro (por ejemplo, por clase social) u horizontalmente (por ejemplo, en la división del trabajo). En nuestro caso la distinción del buen y el mal alumno pone en un lugar distinto al que conoce y reconoce las características del buen alumno, en un lugar de legitimidad.

Por último, son los propios niños y niñas del estudio que dan cuenta de la vivencia hegemónica del neoliberalismo,reproduciendo en parte ese discurso, pero defendiendo, a la vez, un lugar que podríamos llamar "propio”. Hay unaconstante convivencia de mundos opuestos, que incluye en los niños una constante resistencia a los discursos que destruyen toda posibilidad de oposición y crítica.Estos argumentos, muchas veces de carácter moral, los destituyen a ellos y ellas mismas como agentes. No obstante eso, acompañando a la visión casi siempre negativa de las condiciones de pobreza, hay una repetición casi automática del discurso del buen estudiante. Este último funcionaría como un dispositivo efectivo contra la desigualdad, abriendo espacio a la posibilidad del individuo por sobre el sistema.Poner en duda estos discursos por parte de los niños ha significado integrar elementos de sus comunidades como las historias familiares de educación y trabajo, narraciones de gente que conocen, de otros que vieron por televisión. ¿Se puede ser "buen alumno" si no hay condiciones materiales y sociales para ello? ha sido una reflexión que los niños han hecho, oscilando entre disensos y consensos propios de una reflexión crítica.

Darle un lugar en el discurso educativo a aquellos y aquellas estudiantes que no cumplen con los requisitos del buen estudiante, que no tienen familias que hacen elecciones afectivas y/o racionales correctas, pero que son una otredad tan competente como aquella que sí responde a esos requerimientos, es la primera tarea que nos cabe como educadores. Una forma de enfrentar la discriminación es aceptando, justamente, que las competencias educacionales han sido repartidas de manera desigual, por clase social. Si bien los decididores por ahora son hombres, blancos, heterosexuales y de clase media, es nuestra tarea abrir las puertas para que la pobreza no siga reproduciéndose a través de la exclusión de todos y todas los que no cumplen con esos requisitos.

Declarar como agentes sociales críticos competentes a los niños y niñas de las escuelas debería ser la primera tarea. 
Polis, Revista Latinoamericana, Volumen 15, $N^{\circ}$ 45, 2016

\section{Notas}

${ }^{1}$ Artículo realizado en el marco del Proyecto FONDECYT Nº11130092 “Jóvenes vulnerables como analistas críticos de discurso: Un estudio participativo sobre sus propios aprendizajes”.

${ }^{2}$ LEY NÚM. 20.248 "Fija normas para determinar los alumnos con necesidades educativas especiales que serán beneficiarios de las subvenciones para educación especial” 14 Mayo 2009 http://www.mineduc.cl/usuarios/convivencia_escolar/doc/ 201103050142030.Ley_N_20248_Ley_de_Subvencion_Escolar_Preferencial.pdf y DECRETO 170 "Fija normas para determinar los alumnos con necesidades educativas especiales que serán beneficiarios de las subvenciones para educación especial” 25 AGOSTO DEL 2010 HTTP://WWW.LEYCHILE.CL/NAVEGAR?IDNORMA $=1012570$

${ }^{3}$ Ver también a este respecto Álvarez Leguizamón (2008).

${ }^{4} \mathrm{La}$ investigación en curso y de la cuál este trabajo es parte (FONDECYT N¹11300922) es una investigación cualitativa, analítica, que se desarrolla dentro de las lógicas de la investigación - acción con jóvenes "vulnerables" de una escuela municipal y otra rural. Nos interesa, entre otras cosas, dar herramientas críticas a los jóvenes para enfrentar el aprendizaje. Para ello, consideramos a la crítica una "competencia” (Boltanski, 2011, 2012). Más de esto en el apartado de análisis.

${ }^{5}$ 5Programa de Gobierno Michelle Bachelet 2014- 2018 michellebachelet.cl/programa/

${ }^{6}$ www.conacep.cl

${ }^{7}$ Un análisis crítico del concepto de calidad en la educación chilena en Peña (2013). “Análisis Crítico de Discurso del 'Decreto 170' de Subvención Diferenciada para Necesidades Educativas Especiales: El diagnóstico como herramienta de gestión”. Psicoperspectivas, vol. 12, pp. 9-103.DOI:10.5027/PSICOPERSPECTIVAS-VOL 12ISSUE2-FULLTEXT-252

${ }^{8}$ LEY NÚM. 20.609, pg. 1. “Establece medidas contra la discriminación” 24 JULIO 2012. Consultado el 10 de octubre de 2013 en http://www.leychile.cl/ Navegar?idNorma $=1042092$

${ }^{9}$ Según ADIMARK el estrato E o de extrema pobreza se caracteriza por un ingreso familiar de entre USD 359-539, 7,7 años de escolaridad promedio. Un 34\% de la población total pertenece a este estrato, según el Censo del año 2002.

${ }^{10}$ Para fines de este trabajo daremos cuenta de los elementos relacionados con los discursos discriminatorios surgidos de los jóvenes analistas más que de sus competencias. 


\section{Bibliografía}

Abu-Lughod, L. (1990), “The Romance of Resistance: Tracing Transformations of Power Through Bedouin Women”, en American Ethnologist, vol 17, p. 41-55.

ADIMARK (2004), Mapa socioeconómico de Chile. Disponible en: http:/ /www.adimark.cl/medios/estudios/mapa_socioeconomico_de_chile.pdf

Aguirre, M., Adasme, A. y Candia, C. (2009), “Deserción escolar en Chile”, en CEOC Estudios de economía regional en Chile, Centro de estudios de opinión ciudadana, Universidad de Talca, Talca.

Álvarez Leguizamón, S. (2008), Pobreza y desarrollo en América Latina, EUNSA, Salta.

Boltanski, L. (2012), Sociología y crítica social. Ciclo de conferencias en la UDP, Ediciones Diego Portales, Santiago.

Ídem (2010), On critique. A sociology of emancipation, Polity, Cambridge.

Boltanski, L. y Thévenot, L. (1983), “Finding one’s way in social space: a study based on games”, en Social Science Information, vol. 22, pp. 631680 .

Burman, E, (1998), La Deconstrucción de la Psicología Evolutiva, Visor Aprendizaje, Madrid.

Cabalín, C. (2014), “The conservative response to the 2011 Chilean student movement: Neoliberal education and media”, en Discourse: Studies in the Cultural Politics of Education, Vol. 5. http://dx.doi.org/10.1080/ 01596306.2013.871233

Decreto170 "Fija normas para determinar los alumnos con necesidades educativas especiales que serán beneficiarios de las subvenciones para educación especial”, 25 agosto 2010. HTTP://WWW.LEYCHILE.CL/ NAVEGAR?IDNORMA=1012570

Encuesta de Caracterización Socio-Económica Nacional (CASEN) 2009, http://www.ministeriodesarrollosocial.gob.cl/casen2009/educacion.php

Fairclough, N. (2010), Critical discourse analysis. The critical study of language, Longman, London.

Ídem (1995), Critical discourse analysis. The critical study of language. Longman,London.

Ídem (1989), Language and Power, Longman, London. 
Polis, Revista Latinoamericana, Volumen 15, $N^{\circ}$ 45, 2016

Friedman, M. (1995), Public Schools: Make them private. Institute, Washington D.C.DOI: 10.1080/09645299700000026

Harvey, D. (2007), Breve historia del neoliberalismo, Akal, Madrid.

Ketterer, R. (2014), “Reflexiones sobre la reforma educacional”, columna publicada en El Mercurio el 22 de julio del 2014.

Ley Núm. 20.248 “Fija normas para determinar los alumnos con necesidades educativas especiales que serán beneficiarios de las subvenciones para educación especial”, 14 Mayo 2009. http://www.mineduc.cl/usuarios/ convivencia_escolar/doc/201103050142030.Ley_N_20248_Ley_ de_Subvencion_Escolar_Preferencial.pdf

Ley Núm. 20.609 “Establece medidas contra la discriminación”, 24 Julio 2012. http://www.leychile.cl/Navegar?idNorma=1042092

Mouffe, C. (2007), En torno a lo político, Fondo de Cultura Económica, Buenos Aires.

Muñoz León, F. (2013), “No a ‘separados pero iguales’ en Chile: un análisis del Derecho antidiscriminación chileno a partir de su primera sentencia”. Estudios constitucionales, vol. 11, p. 201-228.

Parker, I. (2007), Revolution in Psychology: Alienation to Emancipation, Pluto Press, London.

Ídem (1992), Discourse Dynamics: Critical Analysis for Social and Individual Psychology, Routledge, London.

Peña, M. (2013), “Análisis Crítico de Discurso del 'Decreto 170’ de Subvención Diferenciada para Necesidades Educativas Especiales: El diagnóstico como herramienta de gestión”, en Psicoperspectivas, vol. 12, pp. 9103. DOI: 10.5027/PSICOPERSPECTIVAS-VOL 12-ISSUE2-FULLTEXT252

Ídem (2011), "Privatización de la educación en Chile y sus consecuencias en los sujetos que se educan”, en Polis Revista Latinoamericana, vol. 10, $\mathrm{N}^{\circ} 30$. .

Popham, W. (1999). “¿Por qué las pruebas estandarizadas no miden la calidad educativa?”, en Educational Leadership, vol. 56, $N^{\circ}$ 6, en documento PREAL de marzo de 1999. http://www.oei.es/evaluacioneducativa/ pruebas_estandarizadas_no_miden_calidad_educativa_popham.pdf.

Programa de Gobierno Michelle Bachelet 2014- 2018. michellebachelet.cl/ programa/ 
Redondo, J. (2005), “El experimento chileno en educación. ¿Conduce a mayor equidad y calidad en la educación?”, en Última década, vol. 13, pp. 95 - 110. DOI:

Reisgl, M. y Wodak, R. (2001), Discourse and Discrimination: Rhetorics of Racism, Routledge, London

Rose, N. (2007), The politics of life itself. Biomedicine, power and subjectivity in the twentyfirst century, Princeton University Press, New Jersey.

Ídem (1989), Governing the soul. The shaping of the private self, Routledge, London.

Shapiro, S. (1989), “Educación y Democracia: Estructuración de un discurso contra hegemónico del cambio educativo”, en Journal of Curriculum Theorizing, vol. 8, p. 33 - 54.

Treviño, E., Salazar, F. y Mendoza, F. (2011), “Segregar o incluir, esa no debería ser una pregunta en educación”, en Revista Docencia $\mathrm{N}^{\circ} 45$. p. $34-47$.

Van der Leeuwen, T. (2008), Discourse and Practice, Oxford University Press, New York.

Van Dijk, T. (2003), Ideología y Discurso, Ariel, Madrid.

Wodak, R. (2012), “Discrimination via Discourse: Theories, methodologies and examples”, en Zeitgeschichte, vol. 6, pp. 43-421.

Ídem (2001), “The discourse: an historical approach”, en R. Wodak and M. Meyer (eds.), Methods of critical discourse analysis, Sage, London, p.63-94.

UNICEF - Techo para Chile (2009), “La voz de los niños, niñas y adolescentes en los campamentos.” http://www.unicef.cl/unicef/public/ archivos_documento/348/La\%20Voz\%20Campamento.pdf

Recibido: 27.11.14

Aceptado: 21.09.16 